\title{
A Tablet-based Virtual Environment for Neurosurgery Training
}

Nigel W John ${ }^{1 *}$, Nicholas I Phillips ${ }^{2}$, Llyr ap Cenydd ${ }^{3}$, David Coope ${ }^{4}$, Nick Carleton-Bland ${ }^{5}$, Ian Kamaly-AsI ${ }^{6}$, William P Gray ${ }^{7}$

${ }^{1}$ Department of Computer Science, University of Chester, United Kingdom

${ }^{2}$ Department of Neurosurgery, Leeds General Infirmary, United Kingdom

${ }^{3}$ School of Computer Science, Bangor University, United Kingdom

${ }^{4}$ Wolfson Molecular Imaging Centre, The University of Manchester, United Kingdom

${ }^{5}$ North West Registrar in Neurosurgery, United Kingdom

${ }^{6}$ Neurosurgery, Royal Manchester Children's Hospital, United Kingdom

${ }^{7}$ Neuroscience and Mental Health Research Institute, School of Medicine, Cardiff University, United Kingdom

"corresponding author:

Professor Nigel W John

Department of Computer Science

University of Chester

Thornton Science Park

Pool Lane, Ince

Chester $\mathrm{CH} 2$ 4NU

United Kingdom

nigel.john@chester.ac.uk 


\section{Abstract}

The requirement for training surgical procedures without exposing the patient to additional risk is well accepted and is part of a national drive in the UK and internationally. Computer-based simulations are important in this context, including neurosurgical resident training. The objective of this study is to evaluate the effectiveness of a custom built virtual environment in assisting training of a ventriculostomy procedure. The training tool (called VCath) has been developed as an app for a tablet platform to provide easy access and availability to trainees.

The study was conducted at the first boot camp organized for all year one trainees in neurosurgery in the UK. The attendees were randomly distributed between the VCath training group and the Control group. Efficacy of performing ventriculostomy for both groups was assessed at the beginning and end of the study using a simulated insertion task. Statistically significant changes in performance of selecting the burr hole entry point, the trajectory length and duration metrics for the VCath group, together with a good indicator of improved normalized jerk (representing the speed and smoothness of arm motion), all suggest that there has been a higher level cognitive benefit to using VCath. The app is successful as it is focused on the cognitive task of ventriculostomy, encouraging the trainee to rehearse the entry point and use anatomical landmarks to create a trajectory to the target. In straight-line trajectory procedures such as ventriculostomy, cognitive task based education is a useful adjunct to traditional methods and may reduce the learning curve and ultimately improve patient safety.

\section{Introduction}

The requirement for training surgical procedures without exposing the patient to additional risk is well accepted world-wide and in the UK is now part of a national drive (Consultants Outcomes, 2014). Computer-based simulations are important in this context, including 
neurosurgical resident training (Lemole Jr, Banerjee, Luciano, Neckrysh, and Charbel, 2007;

Schirmer, Elder, Roitberg, and Lobel, 2013). New technologies continue to improve the fidelity of what can be achieved but it is not necessarily a requirement to use expensive hardware and software to produce an effective training tool, as discussed by Aggarwal, Grantcharov, Moorthy, Hance, and Darzi (2006).

Previously we have developed proof of concept training tools for neurosurgery that only require a Web browser and so offer ease of use, any time, from anywhere (Phillips \& John, 2000; John, Riding, Phillips, Mackay, Steineke, et al. 2001). These tools included scenarios to simulate catheterization of the lateral ventricle in the brain, and a lumbar puncture procedure. Moorthy, Jiwanji, Shah, Bello, Munz, et al. (2003) subsequently performed validation studies that demonstrated that these low fidelity environments did improve the training outcomes of medical students.

In recent years, tablet devices, such as the Apple (Cupertino, CA) iPad, have offered a more convenient and portable way of accessing information and communication between teams, and many applications are appearing aimed at clinical professionals (Savel \& Munro, 2011; Zaki \& Doniel, 2014). This paper investigates whether a tablet device can also be used to deliver an effective neurosurgery training tool for a procedure such as ventriculostomy, the insertion of an external ventricular drain (EVD), and tests it's validity as a learning tool that can improve skill. We developed our own tablet-based training tool for an iPad, which has been used in this study and can also be obtained free of charge from Apple's App Store (VCath, 2012). It has been downloaded over 9,000 times at the time of this paper submission. VCath can be considered a low fidelity virtual environment, it utilizes three dimensional computer graphics renderings of a 
virtual patient and surgical tools with real time interaction via the iPad touch panel (see Figure $1)$.

\section{Methods}

The VCath app has been developed for the 3rd generation iPad, which has a dual core A5X processor with quad core graphics and a screen resolution of $2048 \times 1536$ pixels (ap Cenydd, John, Phillips, and Gray, 2012). It also has a touchscreen and so the use of a gesture-based interface becomes possible. The application was coded in $\mathrm{C}++$ and Objective-C using Apple's Xcode integrated development environment. The open source OGRE (Object-Oriented Graphics Rendering Engine) provided all the graphical features required. A Mac mini was used as the development platform, with the working application then being deployed onto an iPad. The introduction of a cannula into a defined body cavity is broken down within VCath into a series of short tasks to determine the entry point, the trajectory, and the depth of insertion. Figure 1 contains four snapshots illustrating VCath in use with the procedure mode selected. In practice mode, the head, skull and brain can be made transparent so that the position of the ventricles can be viewed to give real time visual feedback to the trainees. In procedure mode, transparency is turned off until the task has been completed, Figure 1(c), and metrics are recorded to provide performance feedback to the trainee, Figure 1(d). The metrics are only viewable after the ventricles have been punctures as the number of insertion attempts is used as one of the metrics. All interaction is implemented using finger gestures: the camera view position and zoom can be changed; the positioning of the catheter, its orientation, and its insertion are manipulated be dragging a finger across the tablet screen. 
The first UK neurosurgical boot camp was organized in September 2014 and all year one trainees in neurosurgery in the UK attended. The VCath validation study took place during this event. Twenty five neurosurgery trainees were available for the study, however, four were excluded as they had previously used VCath. The remainder were randomly distributed between the VCath training group (11 members) and the Control group (10 members).

Efficacy of performing ventriculostomy for both groups was assessed at the beginning and end of the study using a simulated insertion task. The "Rowena" neurosurgical physical model (Realistic Operative Workstation for Educating Neurosurgical Apprentices, Delta Surgical Limited, Staffordshire, UK) was used in conjunction with a Brainlab VectorVision (Brainlab AG, Feldkirchen, Germany) neuronavigation system (see Figure 2).

Participants were first tested on their accuracy of siting a burr hole by identifying their preferred site on the left hemicranium while they were blinded to a pre-formed right frontal burr hole. They then used anatomical landmarks based upon their training to insert a catheter through the pre-formed right frontal burr-hole with the aim of positioning the tip of the catheter in a standard location above the Foramen of Monroe (the channels that connect the paired lateral ventricles with the third ventricle at the midline of the brain). Only one attempt was allowed. The ventricular catheter was fitted with a Brainlab pre-calibrated stylet to enable the location and orientation of the catheter to be tracked but participants were blinded from the Brainlab tracking system throughout. Live tracking data was broadcast from the neuronavigation system using the Brainlab VVLink interface to a laptop PC running BioImage Suite 3.0ß4 (Section of Bioimaging Sciences, Yale School of Medicine, USA, www.bioimagesuite.org). It was then relayed as an OpenIGTLink (Tokuda, Fischer, Papademetris, Yaniv, Ibanez et al., 2009) data stream and recorded using the Perk Tutor (Ungi, Sargent, Moult, Lasso, Pinter et al., 2012) training platform 
installed within 3D Slicer 4.3.1 (Fedorov, Beichel, Kalpathy-Cramer, Finetd, Fillion-Robind, et al, 2012). Data from the time the catheter came closest to the center of the burr-hole to the point at which the participant stated they were at the target was analyzed in R statistics 2.11.1 (R Foundation for Statistical Computing, Vienna, Austria). To ensure compatibility between training and testing, the position, scale and orientation of the VCath ventricle model was configured to be as close to the insertion task with the Rowena model as possible, with the placement of the frontal horns being of particular importance. This was achieved by creating appropriate frontal, sagittal and transverse slices of the volume data and using these images to align the anatomy of the VCath virtual patient in a 3D modelling package.

Metrics calculated included the final distance of the catheter tip from an idealized target, time taken to reach the end point, overall distance and the "smoothness" of the trajectory. The idealized target was defined using an older age MRI template (Rorden, Bonilha, Fridriksson, Bender and Karnath, 2012), giving slightly larger ventricles than standard population average templates, fitted to the Rowena head using an iterative affine co-registration algorithm as implemented in Vinci 2.64 (Max-Planck-Institutes für neurologische Forschung, Cologne, Germany) to preserve the ratio of ventricle size to head size. Smoothness of movement of the catheter is derived from the magnitude of jerks in the recorded trajectory where jerk has the standard definition of change in acceleration over time. As this is dependent upon the length of the trajectory, it is normalized for analysis as previously described (Teulings, Contreras-Vidal, Stelmach and Adler, 1997) using:

$$
\text { normalized jerk }=\sqrt{\frac{1}{2} \int \operatorname{jerk}^{2}(t) d t \times \frac{\text { assessed duration }}{\text { assessed length }}{ }^{2}}
$$


Following the initial assessment to establish their naïve skill levels, both groups attended a general lecture on ventriculostomy where they were introduced to the study. At the end of the lecture the VCath group was allowed to familiarize themselves with the VCath app and then selfevaluate their performance over a twenty minute training period using the app's inbuilt metrics. The Control group had no access to VCath.

Both groups were then re-evaluated on the simulated insertion task to further establish their skill level in hitting the ventricles. Our aim is to compare the change in performance of the trainees (between those with exposure to VCath and those in the control group) using the metrics obtained at the pre and post simulated insertion tasks. The Minitab 16 statistical package was used for the analysis.

\section{Results}

No significant difference was found between the two groups at the pretest stage. Probability plots were made of the metrics to determine if it was appropriate to assume that the data values followed a normal distribution. If so, a two-tailed $t$-test was used to determine if the two sets of data were significantly different from each other. If the data values cannot be assumed to be normally distributed, then the Mann-Whitney test was used. The results are presented in Table 1 and Table 2 . 
Table 1: Two Tailed $t$-test (Hypothesis is mean values of VCath Group = mean values of Control Group, alternative hypothesis values are not equal)

\begin{tabular}{|l|l|}
\hline Metric & p-value \\
& (equal variance assumed) \\
\hline Distance from ideal burr hole $(\mathrm{mm})$ & 0.040 \\
\hline Final distance from target $(\mathrm{mm})$ & 0.178 \\
\hline Normalised Jerk & 0.241 \\
\hline Assessed duration (sec) & 0.245 \\
\hline Max assessed depth (mm) & 0.425 \\
\hline Assessed trajectory modulus (mm) & 0.615 \\
\hline
\end{tabular}

Table 2: Two Tailed Mann-Whitney Test (Hypothesis is mean values of VCath Group = mean values of Control Group, alternative hypothesis values are not equal)

\begin{tabular}{|l|l|}
\hline Metric & $\boldsymbol{p}$-value \\
\hline Assessed trajectory length (mm) & 0.1927 \\
\hline Assessed duration (sec) & 0.3072 \\
\hline Assessed start time (sec) & 0.8053 \\
\hline
\end{tabular}

One sample two tailed $t$-tests were then performed for each group to compare the mean difference of the metric values between the tests at the start and end of the study. The results for the metrics with the smallest $p$-values are presented in Tables 3 and 4 . The box plots in Figure 3 
summarize the difference at the start and the end of the day for both groups for those metrics in Tables 3 and 4 with the smallest $p$-values.

Table 3: VCath Group - one sample two tailed $t$-test (comparing mean difference $=0$ )

\begin{tabular}{|l|l|l|}
\hline Metric & p-value & mean \\
\hline Assessed trajectory length $(\mathrm{mm})$ & 0.002 & 25.55 \\
\hline Assessed duration (sec) & 0.024 & 4.5 \\
\hline Distance from ideal burr hole ( $\mathrm{mm})$ & 0.037 & 3.23 \\
\hline Normalised Jerk & 0.085 & 20867 \\
\hline Final distance from target (mm) & 0.176 & 1.97 \\
\hline
\end{tabular}

Table 4: Control Group - one sample two tailed $t$-test (comparing mean difference $=0$ )

\begin{tabular}{|l|l|l|}
\hline Metric & p-value & mean \\
\hline Final distance from target (mm) & 0.006 & 4.59 \\
\hline Assessed duration (sec) & 0.165 & 1.92 \\
\hline Assessed start time (sec) & 0.196 & 1.373 \\
\hline Distance from ideal burr hole (mm) & 0.305 & -2.49 \\
\hline Normalised Jerk & 0.488 & 6287 \\
\hline
\end{tabular}

A graphical representation of the catheter trajectories from the final assessment test is shown in Figure 4. The red paths are from the VCath group, and the black paths are the Control group. A clustering of the red paths near to the ventricles (highlighted in blue) can be observed. 
A questionnaire was also completed by the VCath group at the end of the twenty minute session in which they used the app. Ten of the eleven subjects felt that repeated use of VCath had improved their performance, the final subject did not answer this question. The ratings that they gave are summarised in Table 5.

Table 5: VCath Questionnaire Results showing total numbers for each rating

(1 very easy to 5 difficult)

\begin{tabular}{|c|c|c|c|c|c|}
\hline How did you find the instructions on how to use & 1 & 2 & 3 & 4 & 5 \\
\hline VCath? & 3 & 1 & 1 & 1 & 5 \\
\hline \multirow[t]{2}{*}{ How easy did you find it to reach the target? } & 1 & 2 & 3 & 4 & 5 \\
\hline & & & 8 & 3 & \\
\hline How easy was it to manipulate the VCath models & 1 & 2 & 3 & 4 & 5 \\
\hline on the iPad using finger gestures? & 2 & 1 & 2 & 3 & 3 \\
\hline How useful did you find the metrics displayed by & 1 & 2 & 3 & 4 & 5 \\
\hline VCath on your performance? & 1 & 2 & 2 & 3 & 3 \\
\hline
\end{tabular}

\section{Discussion}

The two tailed tests ( $t$-test and Mann-Whitney) for the hypothesis that the mean values of the metrics obtained by the VCath group are equal to the mean values obtained by the Control group only produced a significant result $(p=0.04)$ for the site selected for the burr hole on the left hemicranium. It was evident that the VCath group improved when compared with the Control group. The $p$-values are not significant for any of the other metrics. The small sample size may mean that the differences were not large enough in this study to be significantly different. The 
next smallest $p$-value is for the final distance from target $(p=0.178)$ and so could be an indicator that this metric would be significant in a larger study.

The one sample $t$-tests carried out for the VCath group show a significant difference between the performance at the start and the end of the study for the assessed trajectory length $(p$ $=0.002)$, assessed duration $(p=0.024)$, and distance from the ideal burr hole entry point $(p=$ 0.037). Although the difference in the normalized jerk is not significant $(p=0.085)$, again the small value is an indicator that this metric could become significant if a larger sample size were used. Note that the performance was in general improving for all of these metrics and the median change in the box plots in Figure 3 (a), (b), (d) and (e) demonstrate a greater improvement in performance for the VCath group than the Control group for these metrics. Also of note from box plot 3D is the wide range in the difference for the assessed trajectory length obtained by the Control group, which suggests a poorer performance than the VCath group.

The only metric that showed a significant difference for the Control group from the one sample $t$-tests is the final distance from target $(p=0.006)$. A small $p$-value is obtained for this metric for the VCath group but as with the two tailed test it is not sufficiently small to be significant. This would indicate that the Control group had a better improvement in performance on this one metric. However, when the catheter trajectories are plotted in a 3D graphical representation (Figure 4) the clustering of the trajectories from the VCath group are more consistently focused on the ventricles. The box plot in Figure 3(c) is also indicating a large overlap in performance between the two groups for this metric.

The questionnaire ratings from the VCath group on the use of the app do not provide any conclusive trends (Table 5). However, the comments made will be taken into account when generating the next version of the VCath app. 


\section{Conclusion}

The purpose of this study was to validate the VCath app as an educational tool for assisting in the training of ventriculostomy. Whereas VCath can only provide a low fidelity simulation of the actual procedure as it is limited by the user interface available on a standard tablet platform, the study has nevertheless indicated that VCath has had a positive effect on performance. Both the VCath and the Control group benefitted from a didactic lecture from an experienced consultant neurosurgeon on how to successfully perform a ventriculostomy. However, the group that received supplementary training with VCath demonstrated a significant improvement when compared to the Control group in selecting the optimum entry point for the burr hole. The statistically significant changes in performance of the trajectory length and duration metrics for the VCath group, together with a good indicator of improved normalized jerk, and the more central clustering of trajectories with VCath, also all suggest that there has been a higher level cognitive benefit to using VCath. The acquisition of the skills required for ventriculostomy has been demonstrated, as has the retention and application of those skills in subsequent performance. The VCath app is successful as it is focused on the cognitive task of ventriculostomy, encouraging the trainee to rehearse the entry point and use anatomical landmarks to create a trajectory to the target. In straight-line trajectory procedures such as ventriculostomy, cognitive task based education is a useful adjunct to traditional methods and may flatten the learning curve and ultimately improve patient safety.

The results obtained from this study will enable us to disseminate the VCath app throughout the neuroscience community as a useful tool in the training curriculum. Future work will include support for other neurosurgery procedures such as trigeminal neuralgia, as well as 
procedures from other surgical specialties. We are also developing a higher fidelity version of

VCath that uses a stylus interface and the option of stereoscopic viewing. A follow on study will be conducted at the 2015 Boot Camp to determine if these factors can improve the training performance.

\section{References}

Aggarwal, R., Grantcharov, T., Moorthy, K., Hance, J., \& Darzi, A. (2006). A competency-based virtual reality training curriculum for the acquisition of laparoscopic psychomotor skill. The American Journal of Surgery, 191(1), 128-133.

Consultant Outcomes Publication, Healthcare Quality Improvement Partnership (2014). Available at: http://www.hqip.org.uk/consultant-outcomes-publication/. Retrieved January 7 2015.

ap Cenydd, L., John, N., Phillips, N., \& Gray, W. (2012). VCath: a tablet-based neurosurgery training tool. Studies in Health Technology and Informatics, 184, 20-23.

Fedorov, A., Beichel, R., Kalpathy-Cramer, J., Finetd, J., Fillion-Robind, J., Pujolaet, S., et al. (2012). 3D Slicer as an image computing platform for the Quantitative Imaging Network. Magnetic Resonance Imaging, 30, 1323-1341.

John, N., Riding, M., Phillips, N., Mackay, S., Steineke, L., Fontaine, B., et al. (2001). Webbased Surgical Educational Tools. Medicine Meets Virtual Reality 2001: Outer Space, Inner Space, Virtual Space, 81, 212-217.

Lemole Jr, G., Banerjee, P., Luciano, C., Neckrysh, S., \& Charbel, F. (2007). Virtual Reality in Neurosurgical Education: PartTask Ventriculostomy Simulation with Dynamic Visual and Haptic Feedback. Neurosurgery, 61(1), 142-149. 
Moorthy, K., Jiwanji, M., Shah, J., Bello, F., Munz, Y., \& Darzi, A. (2003). Validation of a webbased training tool for lumbar puncture. Studies in Health Technology and Informatics, 94, 219-225.

Phillips, N. \& John, N. (2000). Web-based Surgical Simulation for Ventricular Catheterisation. Neurosurgery: Official Journal of the Congress of Neurosurgical Surgeons, 46(4), 933-937.

Rorden, C., Bonilha, L., Fridriksson, J., Bender, B., \& Karnath, H. (2012) Age-specific CT and MRI templates for spatial normalization. Neuroimage, 61, 957-965.

Savel, R., \& Munro, C. (2011). Scalpel, Stethoscope, iPad: The Future Is Now in the Intensive Care Unit. American Journal of Critical Care, 20(4), 275-277.

Schirmer, C., Elder, J., Roitberg, B., \& Lobel, D. (2013). Virtual reality-based simulation training for ventriculostomy: an evidence-based approach. Neurosurgery, 73, S66-S73.

Teulings, H., Contreras-Vidal, J., Stelmach, G., \& Adler, C. (1997). Parkinsonism reduces coordination of fingers, wrist, and arm in fine motor control. Experimental Neurology, 146(1), 159-170.

Tokuda, J., Fischer, G., Papademetris, X., Yaniv, Z., Ibanez, L., Cheng, P., et al. (2009). OpenIGTLink: an open network protocol for image-guided therapy environment. International Journal Medical Robotics and Computer Assisted Surgery, 5, 423-434.

Ungi, T., Sargent, D., Moult, E., Lasso, A., Pinter, C., McGraw, R., et al. (2012) Perk Tutor: an open-source training platform for ultrasound-guided needle insertions. IEEE Transactions on Biomedical Engineering, 59, 3475-3481.

VCath (2012). Available at: https://itunes.apple.com/gb/app/vcath/id568887198. Retrieved January 72015. 
Zaki, M., \& Doniel, D. (2014). Smartphone use in neurosurgery? APP-solutely! Surgical Neurology International, 5, 113.

\section{Acknowledgements}

Jo Mitchell and Marc Edwards from Bangor University for their assistance in organizing the study. Dr Shirley John of the Open University for assistance with the statistical analysis undertaken.

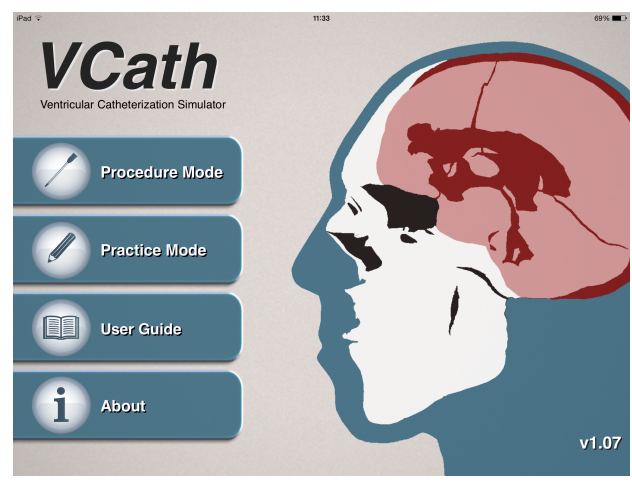

(a)

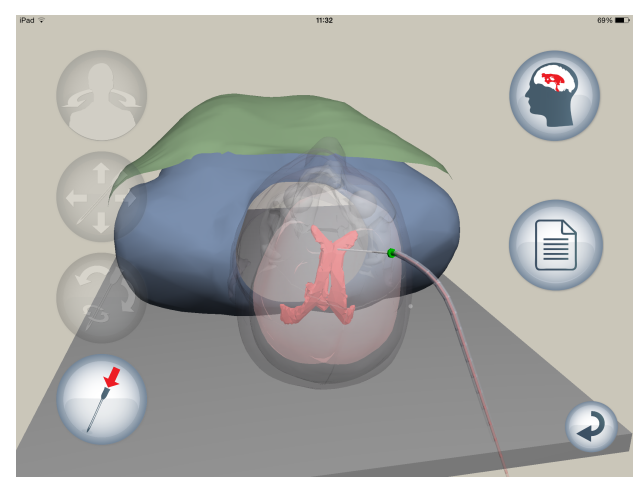

(c)

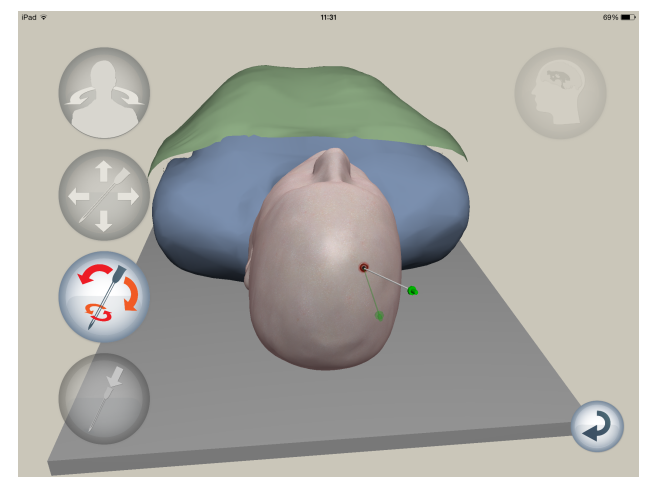

(b)

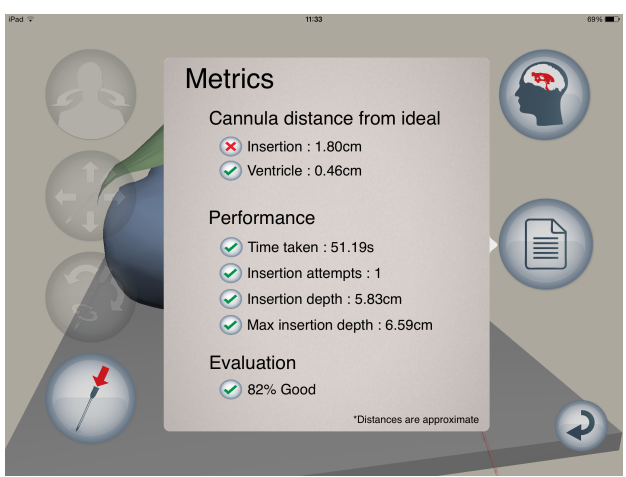

(d)

Figure 1. (a) VCath Menu Screen; (b) The trainee has positioned the virtual patient's head, selected the entry point, and is deciding on orientation of catheter; (c) The catheter has been inserted and punctured the lateral horn of the ventricles - transparency is turned on at end of task; (d) Performance metrics are displayed. 


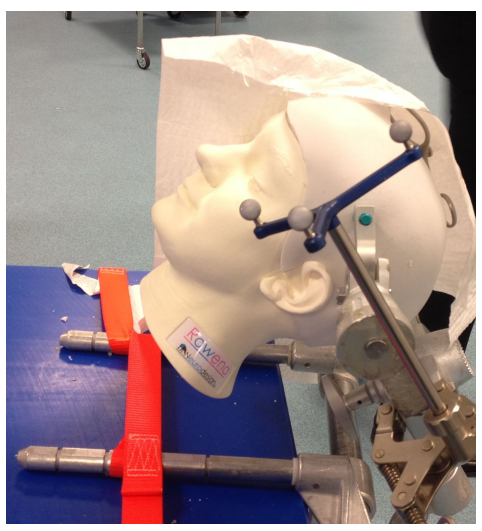

Figure 2: Rowena head model set up for pre- and post-test

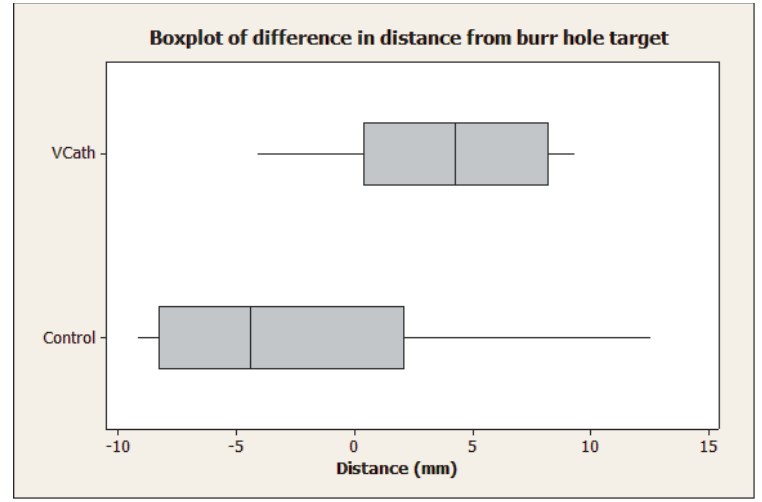

(a)

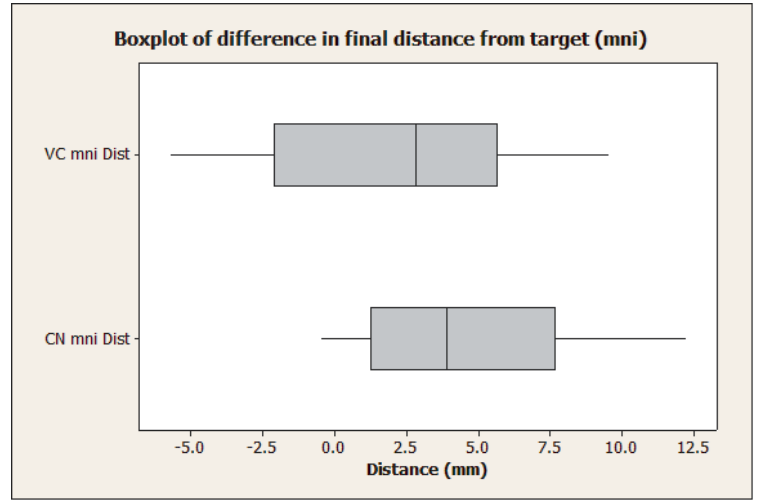

(c)

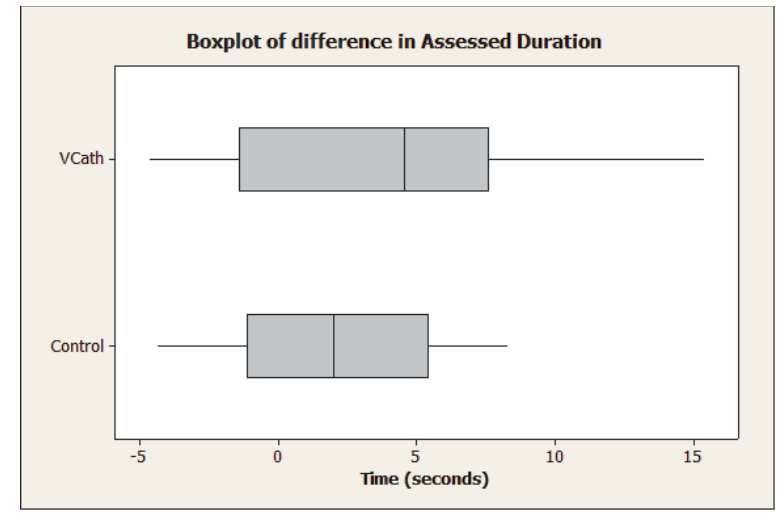

(b)

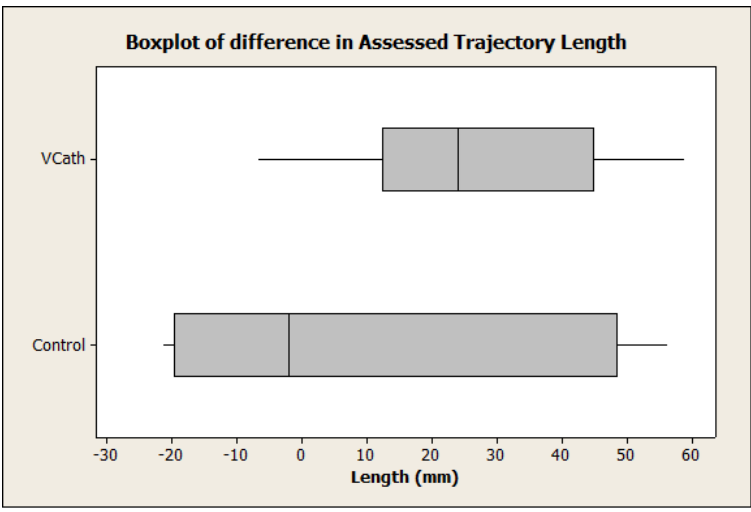

(d) 


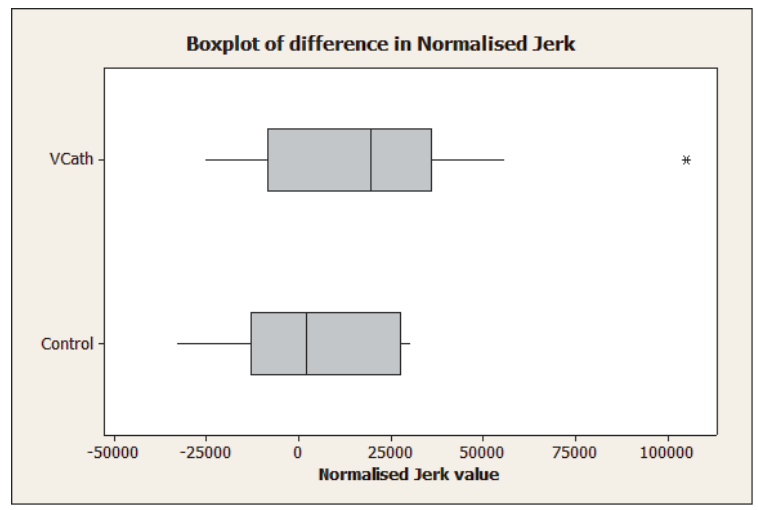

(e)

Figure 3: Box plot representation of the metric differences with small $p$-values. (a) Distance from ideal burr hole entry point; (b) Assessed duration; (c) Final distance from target; (d) Assessed trajectory length; (e) Normalised jerk.

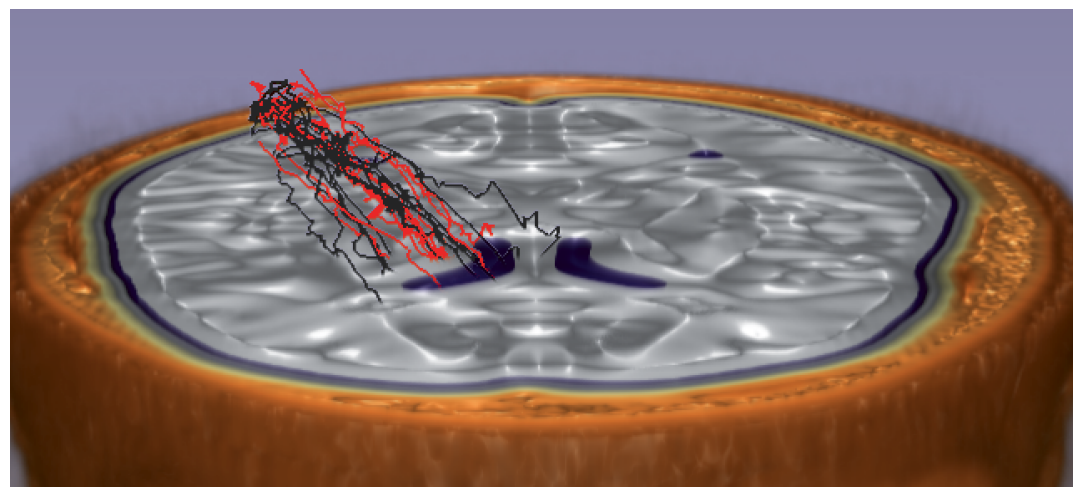

Figure 4: Post training trajectories. The red paths are the VCath group and the black paths are the control group. 